\title{
Localized solutions in Waring's problem: the lower bound
}

\author{
by \\ DiRK DAEMEN (Pforzheim)
}

1. Introduction. We study the classical Waring problem, but try to keep all variables as close together as possible. When $k \geq 2$ and $s$ are fixed, this amounts to counting the number of solutions, say $r_{k, s}(n, Y)$, of the diophantine equation

$$
x_{1}^{k}+x_{2}^{k}+\cdots+x_{s}^{k}=n
$$

subject to the constraints $X-Y<x_{j} \leq X+Y$ for $1 \leq j \leq s$ where

$$
X=\left[\left(\frac{n}{s}\right)^{1 / k}\right]
$$

is the largest integer below the average size of $x_{j}$ in (1.1) and $Y$ is as small as possible.

It is now more than 70 years ago that Wright [6] in an early work showed, inter alia, that for any $\varepsilon>0$ there is an $s_{0}(k, \varepsilon)$ such that whenever $s \geq s_{0}$ and $Y \geq X^{1-1 / k+\varepsilon}$, then $r_{k, s}(n, Y)$ satisfies the asymptotic formula predicted by the Hardy-Littlewood circle method. This has the shape

$$
r_{k, s}(n, Y) \sim c_{\infty} \mathfrak{S}(n) X^{1-k} Y^{s-1},
$$

in our language, where $c_{\infty}>0$ is a certain constant not depending on $n$ and $\mathfrak{S}(n)$ denotes the familiar singular series associated with Waring's problem. One may wonder to what extent (1.3) remains valid when $Y$ takes even smaller values. Note that the right hand side of $(1.3)$ is positive, whence $r_{k, s}(n, Y) \geq 1$ for large $n$. However, Wright [7] also observed that for any fixed $s$ there is a constant $c>0$ letting $r_{k, s}(n, c \sqrt{X})$ vanish on an infinite sequence of natural numbers $n$. Hence, one cannot hope for 1.3 to hold with $Y=c \sqrt{X}$ when $c$ is small.

In the present communication we aim to rekindle interest in this natural variant of Waring's problem by demonstrating that $r_{k, s}(n, Y)$ is, in fact,

Key words and phrases: additive number theory, Hardy-Littlewood method, Weyl sums. 
essentially as large as is predicted by the heuristic asymptotic formula 1.3 . once $Y=c \sqrt{X}$ with a suitably large positive constant $c$.

Theorem. For $j=2,3, \ldots, 10$ define $s_{j}=9,17,47,111,241,415,673$, 1081, 1771 respectively, and for $k>10$ let

$$
s_{k}=2\left[\frac{5 k^{2}}{3} \log k+\frac{29 k^{2}}{30} \log \log k+\frac{7 k^{2}}{3} \log \log \log k+C k^{2}\right]+1
$$

where $C$ is a certain absolute constant so determined that the estimate 4.2 ) below holds. Then there is a positive number $c$, independent of $n$, such that for $Y=c[\sqrt{X}]$ and all $s \geq s_{k}$, one has

$$
r_{k, s}(n, Y) \gg X^{1-k} Y^{s-1} \text {. }
$$

Note that this is the best possible in terms of the constraint on $Y$ by appeal to Wright's result [7]. We remark that by working a little harder, one can replace the 9 for $s_{2}$ by 7 and further that the asymptotic formula 1.3 holds for similar ranges of $s$ as soon as $Y$ only grows a little faster than $\sqrt{X}$. The latter will be shown elsewhere [2], since the procedure is more direct than in this paper. Our proof is based on the circle method with the aid of Vinogradov's familiar mean value theorem and crucially depends on a process that might be called "binomial descent". The said refinement is used both in the derivation of the minor arc estimate 4.5 and in the inductive strategy to verify Lemma 1 in $\S 5$.

Throughout, the notation is standard in number theory and follows Vaughan [4] for the most part.

2. A variation of the main theme. For a natural number $c=c(k, s)$ and $1 \leq j \leq c$, we denote

$$
m=\left[\frac{n-s X^{k}}{k X^{k-1}}\right]-j .
$$

First of all we have $m \ll 1$, since $n-s X^{k} \leq s(X+1)^{k}-s X^{k} \ll$ $X^{k-1}$, by (1.2). But on the other hand, as long as $n=\sum_{j=1}^{s}\left(X+x_{j}\right)^{k}$ with $\left|x_{1}\right|, \ldots,\left|x_{s}\right| \ll \sqrt{X}$, we have

$$
\begin{aligned}
k X^{k-1}\left|\sum_{j=1}^{s} x_{j}\right| & =\left|n-s X^{k}-\sum_{j=1}^{s} \sum_{l=2}^{k}\left(\begin{array}{l}
k \\
l
\end{array}\right) X^{k-l} x_{j}^{l}\right| \\
& \ll\left|n-s X^{k}\right|+\sum_{l=2}^{k} X^{k-l+l / 2} \ll X^{k-1},
\end{aligned}
$$

so that as well $\sum_{j=1}^{s} x_{j} \ll 1$. 
Hence, it seems useful in the case of $Y=c \sqrt{X}$ to let $r_{k, s}(n, m, Y)$ count the number of solutions of the diophantine equations

$$
\sum_{j=1}^{s}\left(X+x_{j}\right)^{k}=n, \quad \sum_{j=1}^{s} x_{j}=m
$$

subject to $-Y<x_{j} \leq Y$ for $1 \leq j \leq s$ where $m$ is given by (2.1). Although $r_{k, s}(n, Y) \geq r_{k, s}(n, m, Y)$, we may expect that $r_{k, s}(n, m, Y)$ only slightly differs from $r_{k, s}(n, Y)$ if $m$ is suitably adjusted.

3. The definition of major and minor arcs. Now the Hardy-Littlewood method comes into play in the computation of $r_{k, s}(n, m, Y)$. We prepare the stage by assuming that $n$ is a large natural number and

$$
\begin{aligned}
& Y=c[\sqrt{X}], \\
& \mathcal{X}=X-Y, \\
& Q=2^{-k} Y^{1 / 3}
\end{aligned}
$$

where the integer $c \geq 4$ will be specified in due course. Further, we define

$$
\mathfrak{M}(q, a)=\left(\frac{1}{4 Q}, 1+\frac{1}{4 Q}\right] \times\left\{\alpha:|\alpha-a / q| \leq \frac{Q}{\mathcal{X}^{k-2} Y^{2}}\right\} .
$$

Then it is easy to verify that when $1 \leq a \leq q \leq Q$ and $(a, q)=1$, the major arcs $\mathfrak{M}(q, a)$ are pairwise disjoint and contained in

$$
U=\left(\frac{1}{4 Q}, 1+\frac{1}{4 Q}\right] \times\left(\frac{Q}{\mathcal{X}^{k-2} Y^{2}}, 1+\frac{Q}{\mathcal{X}^{k-2} Y^{2}}\right] .
$$

We write $\mathfrak{M}$ for the union of the $\mathfrak{M}(q, a)$, and $\mathfrak{m}=\mathcal{U} \backslash \mathfrak{M}$ for the complementary part forming the minor arcs. For brevity, we put $\boldsymbol{\alpha}=\left(\alpha_{1}, \alpha\right) \in \mathbb{R}^{2}$ and, as usual, $e(\alpha)=\exp (2 \pi i \alpha)$, so that short Weyl sums can be denoted by

$$
f(\boldsymbol{\alpha})=\sum_{-Y<x \leq Y} e\left(\alpha_{1} x+\alpha \sum_{j=2}^{k}\left(\begin{array}{l}
k \\
j
\end{array}\right) X^{k-j} x^{j}\right) .
$$

Also, for a measurable set $\mathcal{B} \subset \mathcal{U}$ and $e(\boldsymbol{\alpha})=e\left(-\alpha_{1} m-\alpha\left(n-s X^{k}-\right.\right.$ $\left.k X^{k-1} m\right)$ ), we introduce

$$
\rho(n, m, Y ; \mathcal{B})=\int_{\mathcal{B}} f^{s}(\boldsymbol{\alpha}) e(\boldsymbol{\alpha}) d \boldsymbol{\alpha} .
$$

Finally, on expanding the equation for $n$ in $(2.2)$, we find from the orthogonality that

$$
r_{k, s}(n, m, Y)=\rho(n, m, Y ; \mathfrak{M})+\rho(n, m, Y ; \mathfrak{m}) .
$$


4. Beyond Weyl's inequality. Let $\mathcal{X}$ be given by $(3.2)$. Then, as a first step, we abbreviate

$$
\alpha_{j}=\left(\begin{array}{c}
k \\
j
\end{array}\right) \mathcal{X}^{k-j} \alpha=c_{j} \alpha \quad(2 \leq j \leq k),
$$

so that the modulus of the featured exponential sum (3.5) can be rewritten as

$$
|f(\boldsymbol{\alpha})|=\left|\sum_{1 \leq x \leq 2 Y} e\left(\left(\alpha_{1}-\alpha k\left(X^{k-1}-\mathcal{X}^{k-1}\right)\right) x+\alpha_{2} x^{2}+\cdots+\alpha_{k} x^{k}\right)\right|,
$$

because we intend to compare various approximations to $\alpha_{j}$ for $2 \leq j \leq k$. Some more notation is required. Let $J_{s}^{(k)}(Y)$ count the number of solutions of the diophantine equations

$$
\sum_{r=1}^{s} x_{r}^{j}=\sum_{r=1}^{s} y_{r}^{j} \quad(1 \leq j \leq k)
$$

subject to $1 \leq x_{r}, y_{r} \leq Y$ for $1 \leq r \leq s$. Then Theorem 5.2 of Vaughan [4] implies that, whenever there are $j, a_{j}, q_{j}$ with $2 \leq j \leq k,\left(a_{j}, q_{j}\right)=1$, $q_{j} \leq(2 Y)^{j}$ and $\left|\alpha_{j}-a_{j} / q_{j}\right| \leq q_{j}^{-2}$, we have

$$
f(\boldsymbol{\alpha}) \ll\left(J_{s}^{(k-1)}(Y) Y^{k(k-1) / 2}\left(q_{j}^{-1}+Y^{-1}+q_{j} Y^{-j}\right)\right)^{1 / 2 s} \log Y .
$$

From Theorem 7.4 and the associated remark in [4] and Theorem 7 in [3] we extract the estimates

$$
J_{\left(s_{k}-1\right) / 2}^{(k)}(Y) \ll Y^{s_{k}-1-k(k-1) / 2+\varepsilon},
$$

and thus obtain

$$
f(\boldsymbol{\alpha}) \ll Y^{1+\varepsilon}\left(q_{j}^{-1}+Y^{-1}+q_{j} Y^{-j}\right)^{1 /\left(s_{k-1}-1\right)} .
$$

For $\alpha \in \mathfrak{m}$ it is now feasible to start up the procedure by choosing $a_{j}, q_{j}$ for $j=2, \ldots, k$ with $\left(a_{j}, q_{j}\right)=1,1 \leq q_{j} \leq Y^{j} / Q$ and $\left|\alpha_{j} q_{j}-a_{j}\right| \leq Q Y^{-j}$. Next, suppose that

$$
\left|\alpha_{j} q_{j}-a_{j}\right| \leq Q Y^{-j}, \quad 1 \leq q_{j} \leq Q \quad \text { for } j=2, \ldots, k .
$$

Then, as we shall see below, we would have as well

$$
\left|\alpha-a_{k} / q_{k}\right| \leq Q\left(\mathcal{X}^{k-2} Y^{2}\right)^{-1} \text {. }
$$

Since $\alpha \in \mathfrak{m} \subset \mathcal{U}$, it follows that $1 \leq a_{k} \leq q_{k} \leq Q$, whence, by (3.4), $\alpha$ lies in $\mathfrak{M}$. But this contradicts the definition of the minor arcs. Hence, 4.3 is impossible for $\alpha \in \mathfrak{m}$, and there is always some $j$ such that $Q<q_{j} \leq$ $Y^{j} / Q$. By appeal to the aforementioned bounds and (3.3), therefore, we may provide satisfactory minor arc estimates for $f$, which, at least for $k>2$, are superior to those coming from Weyl's inequality, namely, we obtain

$$
f(\boldsymbol{\alpha}) \ll Y^{1-1 / 3 s_{k-1}} \quad \text { for } \alpha \in \mathfrak{m} .
$$


To confirm (4.4), we prove by induction on $l \in\{0, \ldots, k-2\}$ that

$$
\left|\alpha-a_{k} / q_{k}\right| \leq Q\left(\mathcal{X}^{l} Y^{k-l}\right)^{-1} .
$$

The statement is trivially true for $l=0$, because of 4.3 for $j=k$ and $\alpha_{k}=\alpha$. We assume that (4.6) holds for a certain value of $l$ with $0 \leq l \leq k-3$ and have to show that it also holds for $l+1$. We derive from (4.1) that

$$
\left|c_{k-(l+1)} \frac{a_{k}}{q_{k}}-\frac{a_{k-(l+1)}}{q_{k-(l+1)}}\right| \leq\left(\begin{array}{c}
k \\
l+1
\end{array}\right) \mathcal{X}^{l+1}\left|\alpha-\frac{a_{k}}{q_{k}}\right|+\left|\alpha_{k-(l+1)}-\frac{a_{k-(l+1)}}{q_{k-(l+1)}}\right| .
$$

Then on multiplying the inequality with $q_{k} q_{k-(l+1)}$, and making use of (4.3), it follows from the inductive hypothesis and the definition of $\mathcal{X}$ that

$$
\begin{aligned}
q_{k} q_{k-(l+1)}\left|c_{k-(l+1)} \frac{a_{k}}{q_{k}}-\frac{a_{k-(l+1)}}{q_{k-(l+1)}}\right| & \leq \frac{\left(\begin{array}{c}
k \\
l+1
\end{array}\right) \mathcal{X}^{l+1} Q^{3}}{\mathcal{X}^{l} Y^{k-l}}+\frac{Q^{2}}{Y^{k-(l+1)}} \\
& \leq \frac{2^{k} X Q^{3}}{Y^{3}}+\frac{Q^{2}}{Y^{2}} .
\end{aligned}
$$

In view of (3.1) and (3.3), however, the right hand side is less than $1 / 2$, whence $c_{k-(l+1)} a_{k} q_{k-(l+1)}=a_{k-(l+1)} q_{k}$. This inserted into 4.3) for $j=$ $k-(l+1)$ furnishes proof of 4.6) in the case $l+1$, on noting (4.1).

5. A mean value theorem. We realize that there is an interplay between mean value estimates for $f$ and the number of solutions reflected in $J_{s}^{(k)}(Y)$.

Lemma 1. For every natural number $s$ and any $Y$ satisfying (3.1), we have

$$
\int_{U}|f(\boldsymbol{\alpha})|^{2 s} d \boldsymbol{\alpha} \ll X^{2-k} Y^{k(k+1) / 2-3} J_{s}^{(k)}(Y) .
$$

Proof. In preparation, we consider the integral in terms of its underlying diophantine equation in order to produce an alignment with $J_{s}^{(k)}(Y)$; we are then led to intrinsically more efficient counting functions, say $I_{s}^{(h)}(Y)$, that for $h \in\{1, \ldots, k-1\}$ determine the number of solutions of

$$
\begin{aligned}
& \sum_{r=1}^{s}\left(X+x_{r}\right)^{k}=\sum_{r=1}^{s}\left(X+y_{r}\right)^{k}, \\
& \sum_{r=1}^{s} x_{r}^{j}=\sum_{r=1}^{s} y_{r}^{j} \quad(1 \leq j \leq h)
\end{aligned}
$$

subject to the constraints $-Y<x_{r}, y_{r} \leq Y$ for $1 \leq r \leq s$. With the plausible estimate $I_{s}^{(k-1)}(Y) \ll J_{s}^{(k)}(Y)$ resulting from some minor calculations, it is now apparent that Theorem 3 will follow once the bound

$$
I_{s}^{(1)}(Y) \ll X^{2-k} Y^{k(k+1) / 2-3} I_{s}^{(k-1)}(Y)
$$


is established. For this it suffices to show by induction on $h \in\{1, \ldots, k-1\}$ that

$$
I_{s}^{(1)}(Y) \ll Z(h) I_{s}^{(h)}(Y)
$$

where

$$
Z(1)=1 \quad \text { and } \quad Z(h)=\frac{Y^{3}}{X} \cdots \frac{Y^{h+1}}{X} \quad \text { for } h \geq 2 .
$$

The case $h=1$ is evident. Suppose that $(5.3)$ is true for a particular index $h$ with $1 \leq h \leq k-2$ and verify the same for $h+1$.

To have at hand a simple notation, whenever $h \leq k-2$, we write $I_{s}^{(h)}(Y ; c)$ for the number of solutions captured by $I_{s}^{(h)}(Y)$ under the additional condition $\sum_{r=1}^{s}\left(x_{r}^{h+1}-y_{r}^{h+1}\right)=c$ and remark, for ease of later conclusions, that this is counted by the integral

$\int_{[0,1]^{h+2}}\left|\sum_{x=1-Y}^{Y} e\left(\alpha_{k}(X+x)^{k}+\alpha_{h+1} x^{h+1}+\cdots+\alpha_{1} x\right)\right|^{2 s} e\left(\alpha_{h+1} c\right) d \alpha_{k} \alpha_{h+1} \ldots \alpha_{1}$.

Now extracting from (5.1) and (5.2) for $j=1, \ldots, h$ the identity

$$
\left(\begin{array}{c}
k \\
h+1
\end{array}\right) X^{k-(h+1)} \sum_{r=1}^{s}\left(y_{r}^{h+1}-x_{r}^{h+1}\right)=\sum_{m=h+2}^{k}\left(\begin{array}{c}
k \\
m
\end{array}\right) X^{k-m} \sum_{r=1}^{s}\left(x_{r}^{m}-y_{r}^{m}\right),
$$

and on recalling $-Y<x_{r}, y_{r} \leq Y$ for $1 \leq r \leq s$, we may deduce that

$$
\left|\sum_{r=1}^{s}\left(x_{r}^{h+1}-y_{r}^{h+1}\right)\right| \ll \frac{Y^{h+2}}{X} .
$$

In particular, the right hand side is greater than 1 for $h \geq 1$, by assumption (3.1). Then it follows in sequence from the inductive hypothesis, the triangle inequality and the definition of $Z(h)$ that for some $C \ll Y^{h+2} / X$,

$$
I_{s}^{(0)}(Y) \ll Z(h) I_{s}^{(h)}(Y) \leq Z(h) \sum_{|c| \leq C} I_{s}^{(h)}(Y ; c) \ll Z(h+1) I_{s}^{(h)}(Y ; 0),
$$

as claimed in $(5.3)$ for the case $h+1$, since $I_{s}^{(h)}(Y ; 0)=I_{s}^{(h+1)}(Y)$. This completes the proof.

We apply 4.2 to restate Lemma 1 at once in a more condensed form.

Lemma 2. For $s \geq s_{k}$ and any $Y$ satisfying (3.1), we have

$$
\int_{U}|f(\boldsymbol{\alpha})|^{s-1} d \boldsymbol{\alpha} \ll X^{1-k} Y^{s-2+\varepsilon} .
$$


6. Auxiliary investigations on the generating function. At this point, the generating function (3.5) is expressed in the more fitting form as

$$
f(\boldsymbol{\alpha})=\sum_{-Y<x \leq Y} e\left(\alpha_{1} x+\alpha F(x)\right)
$$

where

$$
F(r)=\sum_{j=2}^{k}\left(\begin{array}{l}
k \\
j
\end{array}\right) X^{k-j} r^{j}
$$

so that an asymptotic expansion to $f$ which is necessary for the evaluation of the major arc contribution may be derived by means of the auxiliary functions

$$
S(q, \boldsymbol{a})=S\left(q, a_{1}, a_{k}\right)=\sum_{r=1}^{q} e\left(\left(a_{1} r+a_{k} F(r)\right) / q\right)
$$

and

$$
I(\boldsymbol{\beta})=I\left(\beta_{1}, \beta_{k}\right)=\int_{-Y}^{Y} e\left(\beta_{1} \gamma+\beta_{k} F(\gamma)\right) d \gamma .
$$

Rather than follow the familiar trail arranging the summation in 6.1 into residue classes to recover by partial summation Theorem 7.1 of Vaughan [4], we produce better error terms by using the Poisson summation formula as given by Vaughan's Lemma 4.2 in [4].

Suppose that $M<N, \psi^{\prime \prime}$ exists, is continuous on $[M, N]$ and $\psi^{\prime}$ is monotonic on $[M, N]$. Let $H_{1}, H_{2}$ denote integers such that $H_{1} \leq \psi^{\prime}(\alpha) \leq$ $H_{2}$ for any $\alpha \in[M, N]$. Then, for $H=\max \left(\left|H_{1}\right|,\left|H_{2}\right|\right)$,

$$
\sum_{M<x \leq N} e(\psi(x))=\sum_{h=H_{1}}^{H_{2}} \int_{M}^{N} e(\psi(\alpha)-\alpha h) d \alpha+O(\log (2+H)) .
$$

This will pave the way for the announced result which is of some independent interest.

Lemma 3. Suppose that $\alpha_{1}=a_{1} / q_{1}+\beta_{1}, \alpha=a_{k} / q_{k}+\beta_{k}$ and $q=\left[q_{1}, q_{k}\right]$. Define $A_{j}=a_{j} q_{j}^{-1} q$ for $j=1$ or $k$ and $\boldsymbol{A}=\left(A_{1}, A_{k}\right)$. Further, assume that

$$
\left|\alpha_{1}-\frac{a_{1}}{q_{1}}\right| \leq \frac{1}{4 q} \quad \text { and } \quad\left|\alpha-\frac{a_{k}}{q_{k}}\right| \leq \frac{1}{4 k^{2} q X^{k-2} Y} \text {. }
$$

Then we have the approximation

$$
f(\boldsymbol{\alpha})=q^{-1} S(q, \boldsymbol{A}) I(\boldsymbol{\beta})+O\left(q^{1+\varepsilon}\right) .
$$

Proof. For convenience, we write

$$
G(\gamma)=\beta_{1} \gamma+\beta_{k} F(\gamma)
$$


as well as

$$
S\left(q, A_{1}, A_{k}, b\right)=\sum_{m=1}^{q} e\left(q^{-1}\left(\left(A_{1} q+b\right) m+A_{k} F(m)\right)\right)
$$

and note the orthogonality $q^{-1} \sum_{-q / 2<b \leq q / 2} e(b(m-x) / q)=1$ for $m \equiv$ $x \bmod q$ and $=0$ else to find the equations

$$
\begin{aligned}
f(\boldsymbol{\alpha}) & =\sum_{m=1}^{q} \sum_{\substack{-Y<x \leq Y \\
x \equiv m \bmod q}} e(G(x)) e\left(a_{1} x / q_{1}+a_{k} F(x) / q_{k}\right) \\
& =q^{-1} \sum_{-q / 2<b \leq q / 2} \sum_{-Y<x \leq Y} e(G(x)-b x / q) S\left(q, A_{1}, A_{k}, b\right) .
\end{aligned}
$$

Hence, the trivial bound $\left|S\left(q, A_{1}, A_{k}, b\right)\right| \leq q$ together with 6.3 reveals that

$$
\left|f(\boldsymbol{\alpha})-q^{-1} S(q, \boldsymbol{A}) \sum_{-Y<x \leq Y} e(G(x))\right| \leq \sum_{\substack{-q / 2<b \leq q / 2 \\ b \neq 0}}|H(b)|
$$

where

$$
H(b)=\sum_{-Y<x \leq Y} e(G(x)-b x / q) .
$$

Now let $-q / 2<b \leq q / 2$ and split $[-Y, Y]$ into finitely many intervals $I$ where $G(\gamma)-b \gamma / q$ is monotonic. Next, 6.6 ensures that

$$
\begin{aligned}
\left|\beta_{1}+\beta_{k} F^{\prime}(\gamma)\right| & \leq \frac{1}{4 q}+\left(4 q k^{2} X^{k-2} Y\right)^{-1} \sum_{j=2}^{k}\left(\begin{array}{c}
k \\
j
\end{array}\right) X^{k-j} j Y^{j-1} \\
& \leq \frac{1}{4 q}+\frac{2\left(\begin{array}{c}
k \\
2
\end{array}\right) X^{k-2} Y+O\left(X^{k-3} Y^{2}\right)}{4 q k^{2} X^{k-2} Y} \\
& \leq \frac{1}{4 q}+\frac{(k(k-1)+o(1)) X^{k-2} Y}{4 q k^{2} X^{k-2} Y} \leq \frac{1}{2 q}
\end{aligned}
$$

Thus the expression $(G(\gamma)-b \gamma / q)^{\prime}=\beta_{1}+\beta_{k} F^{\prime}(\gamma)-b / q$ lies between $-(b+1 / 2) / q$ and $-(b-1 / 2) / q$, whence 6.5 can be applied with $H_{1}=-1$ and $H_{2}=1$ to yield

$$
\sum_{x \in I} e(G(x)-b x / q)=\sum_{h=-1}^{1} \int_{I} e(G(\gamma)-b \gamma / q-\gamma h) d \gamma+O(1)
$$

Consequently, on summing over $I$,

$$
H(b)=\sum_{h=-1}^{1} \int_{-Y}^{Y} e(G(\gamma)-b \gamma / q-\gamma h) d \gamma+O(1)
$$


Since $\left|G^{\prime}(\gamma)-b / q-h\right| \geq\left\|G^{\prime}(\gamma)-b / q\right\| \geq \frac{1}{2}\|b / q\| \geq \frac{1}{2}|b / q|$ for $b \neq 0$ and $|\gamma| \leq Y$, integration by parts yields

$$
\int_{-Y}^{Y} e(G(\gamma)-b \gamma / q-\gamma h) d \gamma \ll|q / b| .
$$

Hence, by (6.8), $H(b) \ll|q / b|$ and, on inserting into (6.7), we obtain

$$
f(\boldsymbol{\alpha})-q^{-1} S(q, \boldsymbol{A}) \sum_{-Y<x \leq Y} e(G(x)) \ll \sum_{\substack{-q / 2<b \leq q / 2 \\ b \neq 0}}|q / b| \ll q^{1+\varepsilon} .
$$

Also $\left|G^{\prime}(\gamma) \pm 1\right| \geq 1 / 2$ for all $|\gamma| \leq Y$ and, by integration by parts, this shows

$$
\int_{-Y}^{Y} e(G(\gamma) \pm \gamma) d \gamma=O(1)
$$

With $b=0$ in $(6.8)$, we finally achieve that

$$
\sum_{-Y<x \leq Y} e(G(x))=\int_{-Y}^{Y} e(G(\gamma)) d \gamma+O(1),
$$

and, in view of 6.9), the desired conclusion follows.

For the sake of completeness, we extract from Theorem 7.3 of Vaughan [4] the bound

$$
I(\boldsymbol{\beta}) \ll Y\left(1+\left|\beta_{1}\right| Y+\left|\beta_{k}\right| X^{k-2} Y^{2}\right)^{-1 / k}
$$

and we learn from his Theorem 7.1 that for $\left(q, a_{1}, a_{k}\right)=1$, one equally has

$$
S\left(q, a_{1}, a_{k}\right) \ll q^{1-1 / k+\varepsilon} .
$$

7. The major arc contribution. Here we profit from our preceding analysis. Indeed, the major arcs are small enough to be treated in a nearly routine way. We define

$$
\mathrm{V}(\boldsymbol{\alpha})= \begin{cases}q^{-1} S(q, \boldsymbol{A}) I\left(\alpha_{1}-a_{1} / q_{1}, \alpha-a_{k} / q_{k}\right) & (\boldsymbol{\alpha} \in \mathfrak{M}), \\ 0 & (\boldsymbol{\alpha} \in \mathfrak{m}) .\end{cases}
$$

Let $\boldsymbol{\alpha} \in \mathfrak{M}$, suppose that $s \geq s_{k}$ and observe the obvious inequality $s \geq$ $3 k+2$. Further we choose $a_{1}, q_{1}$ subject to $\left(a_{1}, q_{1}\right)=1, q_{1} \leq 4 Q$ and $\left|\alpha_{1} q_{1}-a_{1}\right| \leq 1 /(4 Q)$. Then, by (3.3) and (3.4) in combination with $q=$ $\left[q_{1}, q_{k}\right] \leq q_{1} q_{k}$, one has $\left|\alpha_{1}-a_{1} / q_{1}\right| \leq 1 /(4 q)$ and

$$
\left|\alpha-\frac{a_{k}}{q_{k}}\right| \leq \frac{16 k^{2} Q^{3}}{4 k^{2} q_{1} q_{k} \mathcal{X}^{k-2} Y^{2}} \leq \frac{1}{4 k^{2} q X^{k-2} Y} .
$$

We now apply (3.4) and Lemma 3 to readily confirm that

$$
f(\boldsymbol{\alpha})-\mathrm{V}(\boldsymbol{\alpha}) \ll Q^{2+\varepsilon} \quad(\boldsymbol{\alpha} \in \mathfrak{M})
$$


and then detect from 7.1 the crude estimate

$$
\int_{\mathfrak{M}}\left|f^{s}(\boldsymbol{\alpha})-\mathrm{V}^{s}(\boldsymbol{\alpha})\right| d \boldsymbol{\alpha} \ll Q^{2+\varepsilon} \int_{U_{2}}\left(|f(\boldsymbol{\alpha})|^{s-1}+|\mathrm{V}(\boldsymbol{\alpha})|^{s-1}\right) d \boldsymbol{\alpha} .
$$

The definition of $q, A_{1}, A_{k}$ implies that $\left(q, A_{1}, A_{k}\right)=1$ and consequently $S(q, \boldsymbol{A}) \ll q^{1-1 / k+\varepsilon}$, by (6.11). With (6.10), this is used, in view of (7.1), for the integral of $|\mathrm{V}(\boldsymbol{\alpha})|^{s-1}$ to see that

$$
\int_{U_{2}}|\mathrm{~V}(\boldsymbol{\alpha})|^{s-1} d \boldsymbol{\alpha} \ll Y^{s-1} W Z
$$

where

$$
\begin{aligned}
W & =\sum_{q_{1}=1}^{\infty} \sum_{q_{k}=1}^{\infty} q_{1} q_{k} q^{(s-1)(\varepsilon-1 / k)}, \\
Z & =\int_{0}^{\infty} \int_{0}^{\infty} \frac{d \beta_{1} d \beta_{k}}{\left(1+\beta_{1} Y+\beta_{k} X^{k-2} Y^{2}\right)^{(s-1) / k}} .
\end{aligned}
$$

We proceed to estimate $W$ and $Z$. First invoke the definition of $q$ to verify that

$$
\begin{aligned}
W & \ll \sum_{q_{1}=1}^{\infty} \sum_{q_{k}=1}^{\infty} q_{1} q_{k}\left(\frac{q_{1} q_{k}}{\left(q_{1}, q_{k}\right)}\right)^{\varepsilon-3-1 / k}=\sum_{d=1}^{\infty} \sum_{\substack{r_{1}, r_{k}=1 \\
\left(r_{1}, r_{k}\right)=1}}^{\infty} r_{1} r_{k} d^{2}\left(r_{1} r_{k} d\right)^{\varepsilon-3-1 / k} \\
& \ll\left(\sum_{d=1}^{\infty} d^{\varepsilon-1-1 / k}\right)\left(\sum_{r=1}^{\infty} r^{-2}\right)^{2} \ll 1 .
\end{aligned}
$$

Next, observe that for $u, v \geq 0$ one has $(1+u+v)^{-1} \leq(1+u)^{-1 / 2}(1+v)^{-1 / 2}$, whence, by (3.1), we obtain

$$
\begin{aligned}
Z & \ll \int_{0}^{\infty} \frac{d \beta_{1}}{\left(1+\beta_{1} Y\right)^{\frac{s-1}{2 k}}} \int_{0}^{\infty} \frac{d \beta_{k}}{\left(1+\beta_{k} X^{k-2} Y^{2}\right)^{\frac{s-1}{2 k}}} \\
& \ll \int_{0}^{\infty} \frac{d \beta_{1}}{\left(1+\beta_{1} Y\right)^{3 / 2}} \int_{0}^{\infty} \frac{d \beta_{k}}{\left(1+\beta_{k} X^{k-2} Y^{2}\right)^{3 / 2}} \\
& \ll Y^{-1}\left(X^{k-2} Y^{2}\right)^{-1} \ll X^{1-k} Y^{-1} .
\end{aligned}
$$

Inserted into (7.3), in conjunction with $3.3,27.2$ and Lemma 2, this ensures the provisional bound

$$
\int_{\mathfrak{M}}\left|f^{s}(\boldsymbol{\alpha})-\mathrm{V}^{s}(\boldsymbol{\alpha})\right| d \boldsymbol{\alpha} \ll X^{1-k} Y^{s-1-\delta}
$$

where $\delta$ is a suitable positive constant, here and later. Furthermore, by (3.6), we have

$$
\rho(n, m, Y ; \mathfrak{M})=\rho^{*}(n, m, Y ; \mathfrak{M})+O\left(X^{1-k} Y^{s-1-\delta}\right)
$$


where

$$
\rho^{*}(n, m, Y ; \mathfrak{M})=\sum_{q_{1} \leq 4 Q} \sum_{q_{k} \leq Q} \sum_{\substack{a_{1}=1 \\\left(a_{1}, q_{1}\right)=1}}^{q_{1}} \sum_{\substack{a_{k}=1 \\\left(a_{k}, q_{k}\right)=1}}^{q_{k}} \int_{\mathfrak{M}(\boldsymbol{q}, \boldsymbol{a})} \mathrm{V}^{s}(\boldsymbol{\alpha}) e(\boldsymbol{\alpha}) d \boldsymbol{\alpha}
$$

and

$$
\mathfrak{M}(\boldsymbol{q}, \boldsymbol{a})=\left\{\alpha_{1}:\left|\alpha_{1}-a_{1} / q_{1}\right| \leq \frac{1}{4 q_{1} Q}\right\} \times\left\{\alpha:\left|\alpha-a_{k} / q_{k}\right| \leq \frac{Q}{\mathcal{X}^{k-2} Y^{2}}\right\} .
$$

Now we define

$$
S\left(q_{1}, q_{k}\right)=\sum_{\substack{a_{1}=1 \\\left(a_{1}, q_{1}\right)=1}}^{q_{1}} \sum_{\substack{a_{k}=1 \\\left(a_{k}, q_{k}\right)=1}}^{q_{k}}\left(q^{-1} S(q, \boldsymbol{A})\right)^{s} e\left(a_{1} / q_{1}, a_{k} / q_{k}\right)
$$

to rewrite $\rho^{*}(n, m, Y ; \mathfrak{M})$, with reference to 3.4 and $(7.1)$, in the form of

$$
\rho^{*}(n, m, Y ; \mathfrak{M})=\mathfrak{S}^{*}(n, m) J^{*}(n, m)
$$

where

$$
\begin{aligned}
\mathfrak{S}^{*}(n, m) & =\sum_{q_{1} \leq 4 Q} \sum_{q_{k} \leq Q} S\left(q_{1}, q_{k}\right), \\
J^{*}(n, m) & =\int_{-Q /\left(\mathcal{X}^{k-2} Y^{2}\right)} \int_{-1 /\left(4 q_{1} Q\right)}^{1 /\left(4 q_{1} Q\right)} I(\boldsymbol{\beta})^{s} e\left(\beta_{1}, \beta_{k}\right) d \beta_{1} d \beta_{k} .
\end{aligned}
$$

For the completion of $\mathfrak{S}^{*}(n, m)$, we introduce the singular series

$$
\mathfrak{S}(n, m)=\sum_{q_{1}=1}^{\infty} \sum_{q_{k}=1}^{\infty} S\left(q_{1}, q_{k}\right)
$$

and infer, as before, from 6.11 that

$$
\begin{aligned}
\mathfrak{S}(n, m) & =\sum_{d=1}^{\infty} \sum_{\substack{r_{1}, r_{k}=1 \\
\left(r_{1}, r_{k}\right)=1}}^{\infty} \frac{r_{1} r_{k} d^{2}}{\left(r_{1} r_{k} d\right)^{3+2 / k-\varepsilon}} \\
& \ll\left(\sum_{d=1}^{\infty} d^{\varepsilon-1-2 / k}\right)\left(\sum_{r=1}^{\infty} r^{-2}\right)^{2} \ll 1 .
\end{aligned}
$$

Hence, $\mathfrak{S}(n, m)$ is absolutely convergent, uniformly in $n, m$, and thus we have

$$
\begin{aligned}
\mathfrak{S}(n, m)-\mathfrak{S}^{*}(n, m) & \ll \sum_{q_{1} \geq 1} \sum_{q_{k} \geq Q} q_{1} q_{k} q^{\varepsilon-s / k}+\sum_{q_{1} \geq 4 Q} \sum_{q_{k} \geq 1} q_{1} q_{k} q^{\varepsilon-s / k} \\
& \ll \sum_{d=1}^{\infty} d^{\varepsilon-1-2 / k}\left(\frac{d}{Q}\right)^{1 / k}\left(\sum_{r=1}^{\infty} r^{1 / k-2}\right)^{2} \ll Q^{-1 / k} .
\end{aligned}
$$


In just the same manner, we complete $J^{*}(n, m)$ and write the singular integral

$$
J(n, m)=\iint_{\mathbb{R}^{2}} I(\boldsymbol{\beta})^{s} e\left(\beta_{1}, \beta_{k}\right) d \beta_{1} d \beta_{k} .
$$

By 6.10$)$, we then reach the auxiliary bounds

$$
J(n, m) \ll Y^{s} \int_{0}^{\infty} \frac{d \beta_{1}}{\left(1+\beta_{1} Y\right)^{3 / 2}} \int_{0}^{\infty} \frac{d \beta_{k}}{\left(1+\beta_{k} X^{k-2} Y^{2}\right)^{3 / 2}} \ll Y^{s-1} X^{1-k}
$$

and

$$
\begin{aligned}
J(n, m)-J^{*}(n, m) \ll & Y^{s} \int_{\left(16 Q^{2}\right)^{-1}}^{\infty} \frac{d \beta_{1}}{\left(1+\beta_{1} Y\right)^{3 / 2}} \int_{0}^{\infty} \frac{d \beta_{k}}{\left(1+\beta_{k} X^{k-2} Y^{2}\right)^{3 / 2}} \\
& +Y^{s} \int_{0}^{\infty} \frac{d \beta_{1}}{\left(1+\beta_{1} Y\right)^{3 / 2}} \int_{Q / \mathcal{X}^{k-2} Y^{2}}^{\infty} \frac{d \beta_{k}}{\left(1+\beta_{k} X^{k-2} Y^{2}\right)^{3 / 2}} \\
\ll & Y^{s-1-1 / 6} X^{2-k} Y^{-2} \ll X^{1-k} Y^{s-1-1 / 6} .
\end{aligned}
$$

Finally, on collecting together, we may conclude that

$$
\rho(n, m, Y ; \mathfrak{M})=\mathfrak{S}(n, m) J(n, m)+O\left(X^{1-k} Y^{s-1-\delta}\right) .
$$

8. The singular integral. Here, the expression in $(7.6)$ is calculated; it turns out that this will produce the bulk of the main term on the right hand side of (1.4). As a first step, we derive from (6.4) that

$$
J(n, m)=Y^{s} \iint_{\mathbb{R}^{2}}\left(\int_{-1}^{1} e\left(\beta_{1} Y \gamma+\beta_{k} F(Y \gamma)\right) d \gamma\right)^{s} e\left(\beta_{1}, \beta_{k}\right) d \beta_{1} d \beta_{k},
$$

whence the substitution $\beta_{1} \rightsquigarrow Y \beta_{1}, \beta_{k} \rightsquigarrow X^{k-2} Y^{2} \beta_{k}$ reveals that

$$
\left.J(n, m)=X^{2-k} Y^{s-3} \iint_{\mathbb{R}^{2}[-1,1]^{s}} \int_{\beta_{1}} e(\gamma)+\beta_{k} v(\boldsymbol{\gamma})\right) d \boldsymbol{\gamma} d \beta_{1} d \beta_{k}
$$

where

$$
u(\gamma)=\sum_{i=1}^{s} \gamma_{i}-\frac{m}{Y} \quad \text { and } \quad v(\gamma)=\sum_{i=1}^{s} \frac{F\left(Y \gamma_{i}\right)}{X^{k-2} Y^{2}}-\frac{n-s X^{k}-k X^{k-1} m}{X^{k-2} Y^{2}} .
$$

Now, for $\gamma=\left(\gamma_{1}, \ldots, \gamma_{s}\right) \in[-1,1]^{s}$, we shall examine the equations

$$
u(\gamma)=v(\gamma)=0,
$$

which, by the definition of $F$, can also be read as

$$
\sum_{i=1}^{s}\left(X+Y \gamma_{i}\right)=s X+m, \quad \sum_{i=1}^{s}\left(X+Y \gamma_{i}\right)^{k}=n .
$$


From these we extract the relation

$$
\left(\begin{array}{l}
k \\
2
\end{array}\right) X^{k-2} Y^{2} \sum_{i=1}^{s} \gamma_{i}^{2}=n-s X^{k}-k X^{k-1} m-\sum_{i=1}^{s} \sum_{j=3}^{k}\left(\begin{array}{l}
k \\
j
\end{array}\right) X^{k-j} Y^{j} \gamma_{i}^{j}
$$

and thus the asymptotics

$$
\sum_{i=1}^{s} \gamma_{i}^{2}=\frac{n-s X^{k}-k X^{k-1} m}{\left(\begin{array}{c}
k \\
2
\end{array}\right) X^{k-2} Y^{2}} \pm O\left(\frac{Y}{X}\right) .
$$

But (2.1) implies $k X^{k-1} \leq n-s X^{k}-k X^{k-1} m \leq k(c+1) X^{k-1}$ and ensures the inequalities

$$
\frac{X}{k Y^{2}}-\frac{1}{4} \leq \sum_{i=1}^{s} \gamma_{i}^{2} \leq \frac{2(c+1) X}{Y^{2}}+\frac{1}{4}
$$

for all large $n$. Since $c \geq 4$, this shows with (3.1) that $\left|\sum_{i=1}^{s} \gamma_{i}^{2}\right| \leq 1$ for sufficiently large $n$, whence $\gamma \in[-1,1]^{s}$. (Observe that this is not necessarily the case if $Y=o(\sqrt{X})$ and that, indeed, only here is the strong restriction on $Y$ needed.) Hence, $(8.2)$ defines an $s-2$-dimensional subspace $L \subset[-1,1]^{s}$ with positive volume. Then applying Fourier's formula

$$
\lim _{R \rightarrow \infty} \int_{-R}^{R} V(t) e(t \lambda) d \lambda=V(0)
$$

twice to the integral in (8.1), and recalling (3.1), we obtain

$$
J(n, m)=X^{2-k} Y^{s-3} \int_{L} d L \gg X^{1-k} Y^{s-1} .
$$

9. The singular series. To examine the singular series $(7.4)$ it is useful to study its close connection to $M_{n, m}(q)$ that counts the number of solutions of the congruences

$$
\begin{aligned}
F\left(x_{1}\right)+\cdots+F\left(x_{n}\right) & \equiv n-s X^{k}-k X^{k-1} m \bmod q, \\
x_{1}+\cdots+x_{n} & \equiv m \bmod q,
\end{aligned}
$$

subject to $1 \leq x_{j} \leq q$ for $1 \leq j \leq s$. In fact, if we note that $q^{-1} S\left(q, A_{1}, A_{k}\right)=$ $\left(q_{1}, q_{k}\right)^{-1} S\left(q_{1} q_{k}, a_{1}, a_{k}\right)$ for $q=\left[q_{1}, q_{k}\right]$, then, by the proof of Lemma 8 of Arkhipov [1] with minor changes, one may readily confirm that for $s>$ $k(k+1) / 2+1$, this yields

$$
\mathfrak{S}(n, m)=\lim _{t \rightarrow \infty} M_{n, m}(t !)(t !)^{2-s} .
$$

Moreover, by his Lemma 9, we have

$$
\mathfrak{S}(n, m)=\prod_{p} \varphi_{p} \quad \text { with } \quad \varphi_{p}=\lim _{t \rightarrow \infty} M_{n, m}\left(p^{t}\right) p^{t(2-s)} .
$$


Since $\mathfrak{S}(n, m)$ converges absolutely, we now deduce from $(9.1)$, in the same manner as in the proof of Theorem 2.4 of Vaughan [4], that there is a positive number $p_{0}=p_{0}(k)$ with

$$
\frac{1}{2}<\prod_{p>p_{0}} \varphi_{p}<\frac{3}{2}
$$

To check that $\mathfrak{S}(n, m)>0$, it suffices therefore to establish that there are a constant $c$ together with an integer $m$ as in (2.1) and for any prime number $p \leq p_{0}$ some $u=u(p)<\infty$ such that for all $t \geq u$ and $s \geq s_{k}$, one has

$$
M_{n, m}\left(p^{t}\right) \geq p^{(t-u)(s-2)} .
$$

To see this, we apply the definition of $M_{n, m}(q)$ and (6.2) to find by a simple transformation that $M_{n, m}(q)$ counts the number of solutions of the system

$$
\begin{aligned}
y_{1}^{k}+\cdots+y_{s}^{k} & \equiv n \bmod q, \\
y_{1}+\cdots+y_{s} & \equiv m+s X \bmod q,
\end{aligned}
$$

subject to $1 \leq y_{j} \leq q$ for $1 \leq j \leq s$. By Lemmas 2.13 and 2.15 of Vaughan [4], we may find for any prime number $p$ some $u=u(p)<\infty$ such that (9.4) has at least $p^{(t-u)(s-1)}$ solutions modulo $p^{t}$ for all $t \geq u$ and $s \geq s_{k}$. Hence, on rearranging the variables if necessary, there is also a solution $\boldsymbol{a}=\left(a_{1}, \ldots, a_{s}\right)$ for which (as a $p$-adic number) $a_{1}^{k-1} \neq a_{2}^{k-1}$. But the Chinese remainder theorem provides an integer $m$ of the form (2.1) such that $\boldsymbol{a}$ is a solution of both (9.4) and (9.5) for all $p \leq p_{0}$. From this the assertion now follows in the same manner as in the proof of Lemma 6.7, case (a) of Wooley [5].

Finally, by (7.5), 9.1), 9.2 and (9.3), there are integers $c$ and $m$ as in (2.1) such that for all $s \geq s_{k}$, we have

$$
1 \ll \mathfrak{S}(n, m) \ll 1 .
$$

10. Proof of the Theorem: Conclusion. Let $s \geq s_{k}$. Then, on combining (4.5) and Lemma 2, we may infer from (3.6) that

$$
\rho(n, m, Y ; \mathfrak{m}) \ll \sup _{\boldsymbol{\alpha} \in \mathfrak{m}}|f(\boldsymbol{\alpha})| \int_{U_{2}}|f(\boldsymbol{\alpha})|^{s-1} d \boldsymbol{\alpha} \ll X^{1-k} Y^{s-1-1 /\left(3 s_{k-1}\right)+\varepsilon} .
$$

Together with (3.7), (7.7), 8.3) and (9.6), this ensures the desired lower bound for $r_{k, s}(n, Y)$.

\section{References}

[1] G. I. Arkhipov, On the Hilbert-Kamke problem, Math. USSR-Izv. 24 (1985), no. 1, 1-47. 
[2] D. Daemen, The asymptotic formula for localized solutions in Waring's problem and approximations to Weyl sums, Bull. London Math. Soc., to appear.

[3] L. K. Hua, Additive Primzahltheorie, Teubner, Leipzig, 1959.

[4] R. C. Vaughan, The Hardy-Littlewood Method, 2nd ed., Cambridge Univ. Press, Cambridge, 1997.

[5] T. D. Wooley, On simultaneous additive equations II, J. Reine Angew. Math. 419 (1991), 141-198.

[6] E. M. Wright, Proportionality conditions in Waring's problem, Math. Z. 38 (1934), $730-746$.

[7] - , The representation of a number as a sum of four 'almost equal' squares, Quart. J. Math. Oxford 8 (1937), 278-279.

Dirk Daemen

Blumenheckstraße 54

D-75177 Pforzheim, Germany

E-mail: d.daemen@web.de

Received on 17.8.2008

and in revised form on 8.12.2009 